\title{
Pediatric Adherence to HIV Antiretroviral Therapy
}

\author{
Jessica Haberer, MD, MS $^{1}$ and Claude Mellins, PhD $^{2}$ \\ ${ }^{1}$ Massachusetts General Hospital Center for Global Health Harvard Institute for Global Health 104 \\ Mt. Auburn Street, $3^{\text {rd }}$ floor Cambridge, MA 02138 Phone (617) 495-9353 Fax (617) 495-8231 \\ jhaberer@partners.org \\ ${ }^{2}$ HIV Center for Behavioral Studies, NewYork State Psychiatric Institute, and Columbia University \\ Box 15, 1051 Riverside Drive New York, NY 10032 Phone (212) 543-5969 Fax (212) 543-6003 \\ cmellins@gmail.com
}

\begin{abstract}
More than two million children are infected with HIV globally. Pediatric ART adherence is complex and current levels are often suboptimal. As established treatment programs in developed settings struggle with chronic therapy and nascent treatment programs in resource-limited settings expand, the importance and challenges of good adherence to antiretroviral therapy are becoming ever more clear. Adherence behavior is influenced by many factors, which may be categorized as characteristics of the child, the caregiver(s) and family, the regimen, and society and culture. Many of these influences complicate measurement of pediatric adherence and there is no gold standard. This article provides a conceptual framework and evidence-based look at the factors influencing ART adherence in children and aims to identify areas for intervention for this vulnerable population in need.
\end{abstract}

\section{Introduction}

The United Nations Joint Program on AIDS (UNAIDS) estimates that over two million children under the age of 15 are living with HIV/AIDS in the world, with nearly $80 \%$ of these children living in sub-Saharan Africa.[1] High levels of antiretroviral therapy (ART) adherence are critical for viral suppression and reduced morbidity and mortality among HIV infected children.[2-3]

Through successful prevention of mother to child transmission (PMTCT) programs, developed countries face few new cases of infant HIV-infection annually; however, as a result of successful ART use, children are surviving into adolescence and struggling with many adherence challenges associated with long-term therapy. Difficulties include high rates of drug resistance due to prior treatment with suboptimal therapy, severe side effects, mental health issues, and horizontal infections among adolescents.[4,5] Developing countries are confronting different challenges for achieving and maintaining ART adherence as they scale-up pediatric ART programs.[6] Healthcare resources are typically constrained and the burden of co-morbid disease is high.

In a recent systematic review of pediatric ART adherence studies in middle- and low-income countries, Vreeman et al [7] found that estimates of ART adherence levels ranged from $49 \%$ to $100 \%$, with $76 \%$ of articles reporting greater than $75 \%$ adherence. The authors contrasted these values with reports from high-income countries, as summarized in another recent

Correspondence to: Jessica Haberer.

Disclosure: No potential conflicts of interest relevant to this article were reported. 
systematic review by Simoni et al.[8] Estimates of pediatric adherence levels from high-income countries ranged even more widely from $20 \%$ to $100 \%$, and 33 of 55 studies report ART adherence less than $75 \%$. The initial high levels of adherence in developing countries are encouraging; however, adherence failures may become increasingly common as healthier children gain access and confront the long-term challenges of adherence to ART. Even with the early success, resistance levels among some children are unacceptably high.[9] Limited availability of second-line therapy emphasizes the importance of adherence and preservation of the first-line regimen.

The goal of this paper is to help HIV clinicians and researchers conceptualize ART adherence behavior in children. It is not a systematic review of adherence studies; rather, this paper provides a framework and an evidence-based look at the factors influencing pediatric ART adherence in both developed and developing settings. It aims to improve the understanding of the complex challenges and to identify potential areas for intervention with this vulnerable population urgently in need of support.

\section{Framework}

Many of the issues affecting adherence to ART are similar to those affecting children with other chronic illnesses, such as asthma, renal disease, diabetes, and cancer.[10,11] These issues include psychiatric illness, psychological factors, health problems, demanding regimens, children's progressing developmental stages, and varying family perspectives and relationships. There are, however, important differences between HIV and other chronic illnesses. For instance, HIV is multigenerational in nature and has a unique social stigma surrounding transmission.[4] Moreover, its epidemiology is frequently associated with low socio-economic status, minority ethnicity, and/or substance abuse.[1,12]

These various factors make ART adherence behavior in children complex, and HIV-infected children represent a very heterogeneous population globally. A horizontally infected 16 yearold taking pills in France will have very different challenges compared with a vertically infected three year-old orphan taking syrups in Uganda. To help conceptualize pediatric ART adherence, it is useful to consider four major influences on HIV-infected children (Figure 1): child characteristics, caregiver and family characteristics, regimen characteristics, and society and cultural characteristics.

\section{Child Characteristics}

This section reviews the child-specific factors that may impact adherence, beginning with the influence of physical and neurologic development. Other significant areas include the child's health and nutrition, treatment fatigue and denial, medication refusal, and knowledge of HIV status.

\section{Developmental stage}

A child's changing abilities and needs, both physical and emotional, create dynamic challenges over time. Infants depend entirely on their caregiver(s) for ART administration, including redosing after emesis, which is common at this age. Challenges for toddlers and preschoolers typically include independence and medication refusal. School-aged children often develop the capacity to understand concepts of illness and the need for treatment; however, they are usually unaware of their infection.[13] These children also typically want to fit in with their peers and may wonder why they are taking medicines every day when other children are not.

Children who have been highly adherent to ART at younger ages frequently have adherence problems during adolescence, a challenging time developmentally even without chronic 
illness. Horizontally infected adolescents similarly have significant adherence problems. The long-term importance of ART adherence may be lost to other shorter-term priorities such as appearance, social/sexual relationships, and overall identity development. Mental health issues are common in adolescents due to chronic illness, stigma, and/or inherited mental illness and may impede adherence.[4] Studies among US adolescents have shown an association between adherence and being in school/having an established routine, lower alcohol use, belief in selfefficacy, and good outcome expectancy with treatment. Conversely, younger age, later stage disease, depression, fear of HIV disclosure and stigma, regimen complexity, adverse effects, forgetfulness, absence of symptomatology, limited knowledge about HIV, and poor providerpatient relationship have all been associated with worse adherence.[13,14]

Studies in the US and Africa have shown that adherence levels generally decline with increasing age among HIV-infected children.[15-17] Reasons for this phenomenon are varied; the most common influencing factors are presented in the following text.

\section{Neurodevelopment}

Neurodevelopmental and cognitive deficits are common in HIV-infected children.[4,18] Neurologic impairment may be caused directly by HIV, including encephalopathy, or may stem from alcohol and drug exposure pre- and post-natally, as is common in many children infected through vertical transmission in developed countries. These children frequently experience delayed development and lack the cognitive skills to fully understand treatment.

\section{Health and nutrition}

The impact of health and nutrition on adherence is not clear. A US study found an association between worse adherence and advanced disease, which was also seen in a qualitative study from Uganda.[14,19] One potential explanation is that caregivers may take care of a child less well, if they believe that child is at high risk for dying. An Ethiopian study also found that children who received nutritional support had worse adherence, presumably reflecting the presence, rather than the treatment, of malnutrition. Another study from Uganda, however, found that increased hospitalization was associated with higher adherence, perhaps reflecting motivation to get well.[20] The difference in these findings may reflect the difficulty in determining if poor health and malnutrition lead to poor adherence, or vice-versa. Food insecurity is another important factor and is discussed below.

\section{Treatment fatigue and denial}

Treatment fatigue after several years of therapy is common and has been shown in children with other chronic illness, such as cancer.[21] Children may ask why they continue to take medicine when they feel well. Physically apparent side effects, such as lipodystrophy from treatment with stavudine, may cause considerable distress.[22] Both vertically and horizontally infected children may experience denial about their infection,[23] and denial has been associated with poor adherence in chronic treatment of pediatric transplant patients.[24]

\section{Knowledge of HIV status}

Disclosure of chronic illness to a child is a controversial and emotional issue that is particularly difficult for HIV given the associated stigma and the potential for guilt in the case of vertical transmission.[13] Pediatric HIV clinicians generally promote disclosure as a positive influence on adherence; however, the evidence from the literature is mixed. Because disclosure typically does not occur until late childhood or adolescence[13], the effects of disclosure may be difficult to distinguish from those of age. In an Italian study of children over eight years, disclosure was associated with worse adherence.[25] Studies in the US and Ethiopia found similar results when controlling for potential covariates.[26,27] No effect, however, was seen for age or 
disclosure on adherence in US and Ugandan studies[20,28]. Another US study similarly found no effect for disclosure, although age was associated with increased non-adherence prior to multivariate analysis.[16] Finally, qualitative studies in Uganda and Belgium found disclosure was generally associated with improved adherence.[29,30]

\section{Psychosocial function}

As noted above, depression and anxiety are commonly associated with adolescence. They are also common in younger children and have been found to influence ART adherence.[4,31] Poor mental health may lead to denial of infection, apathy, and hopelessness, resulting in medication refusal. Child stress has also been associated with poor adherence[15].

Psychosocial function has been given relatively limited attention; however, these issues may be just as critical as biomedical and socioeconomic factors for the success of treatment.[32]

\section{Caregiver and Family Characteristics}

A child's adherence to ART is strongly influenced by caregiver(s) and family function, which may change over time. The caregiver physically gives the medicine to younger children. While this physical role diminishes as the child grows older, the need for caregiver and family support remains critical at every age. The following paragraphs review how key caregiver characteristics, the child-caregiver relationship, and family structure and resources may affect promotion of ART adherence.

\section{Biologic relationship}

Data are conflicting on the impact of the caregiver's biologic relationship to the child. In cases of vertical transmission, the biologic mother must deal with the potential guilt associated with MTCT. Consequent depression and concurrent HIV or other comorbidities may worsen her ability to care for the child. An Italian study[25] found that younger children living with nonbiologic caregivers had better adherence. Similar findings were seen in a US study; however, the association did not maintain significance when controlling for other factors.[26] Conversely, a Romanian study of horizontally infected adolescents found that non-biologic caregivers were associated with worse adherence.[33] A biologic caregiver may have a stronger emotional connection with the child and be more motivated to promote good adherence compared with a non-biologic caregiver. Caregivers who are also on ART may draw from their own experiences to support their child's adherence. These mixed findings suggest that other factors may be more relevant.

\section{Caregiver permanence}

In cases of vertical transmission, the biologic parents may have died or may be too ill to care for the child, who then receives care from one or more relatives, family friends, or government agencies. In developing countries with limited social services, there is often little to no oversight of foster care, and a child may or may not be under the care of someone well equipped to oversee ART adherence.

Caregivers may also change because of other commitments. For instance, a caregiver may need to travel for extended periods for work (e.g. migratory farming, employment opportunities in urban centers). Adherence may suffer during periods of alternate caregivers is particularly vulnerable because of factors such as disrupted routines, potentially poor communication with new relationships, lack of disclosure, and limited adherence counseling and education with the new caregiver. A study of children in Cote d'Ivoire showed that the presence of multiple caregivers was associated with poor adherence.[34] 


\section{Caregiver beliefs}

Caregivers who deny their child's infection are less likely to be invested in adherence. A qualitative Belgian study found that caregivers who accept the child's infection may be more likely to internalize the information received from the clinician and thus develop a stronger motivation to fight for the child's life.[30] In another qualitative study of US mothers, adherence practices were affected positively by mothers' commitment to adherence and negatively by children adopting their mothers' poor attitudes about medications.[35]

\section{Caregiver education and comprehension of ART administration}

Administration of ART can be complex, especially in children who take syrups and change regimens frequently, as is discussed below. Poor knowledge of regimens is common,[35] and a Romanian study found an association between non-adherence and poor caregiver education. [33] This relationship, however, was not significant in a US study.[26] Caregiver cognitive ability has also been shown to be correlate with adherence,[15] and caregivers' perceived difficulty with ART administration was negatively associated with adherence in the US and in Togo, West Africa.[37,38]

\section{Caregiver psychosocial function}

Poor mental health is common in caregivers of HIV-infected children and has been found to influence caregiver support of a child's adherence.[4] In the case of vertical infections, primarily in developed countries, many children were born to mothers with substance abuse histories and prevalent heritable mental illness.[12,31] Environmental stressors due to limited resources combined with the many emotional and physical needs of an HIV-infected child lead to poor psychosocial function.[15]

\section{Disclosure of HIV status to others}

Caregivers in both developed and developing countries have cited stress and inadequate support as potential barriers to adherence.[15,26,38] Disclosure of the child's HIV status, as well as the caregiver's HIV status, if relevant, to family and the community can help reduce stress and mobilize support for adherence. Such social capital has been theorized to affect adherence behavior in adults living in resource-constrained settings[39] and may be relevant for children. A US study found that adherence strategies depended heavily on family support and resolution of disclosure issues in the household.[40] Disclosure to others has been associated with good adherence in both the US[15] and Uganda.[19] However, stigma and discrimination are also known barriers to adherence, [13] and caregivers may therefore be reluctant to disclose a child's HIV status to anyone, even within the household.

Disclosure at school is of particular concern. School nurses or other staff can be useful allies in maintaining adherence, especially when dosing must occur during school hours. A US survey found non-adherent caregivers were less likely to disclose the child's HIV status to the school; [37] however, disclosure of the child's status at school could be detrimental. Many children do not yet know or fully understand the meaning of their HIV infection, and disclosure to the school could result in unintended disclosure to the child, as well as stigma and discrimination from the school staff and students.

\section{The caregiver-child relationship}

The relationship between the caregiver and the child may have a profound influence on adherence. Giving medication is an interactive process that is shaped by the child's behavior and the caregiver's expectations.[35] When communication is poor, adherence has been shown to suffer.[15] Communication may be particularly challenging as youth approach adolescence. 


\section{Responsibility for adherence}

The responsibility for adherence clearly lies with the caregiver for infants and young children. However, as children mature and following disclosure, the responsibility for adherence shifts and adherence may be influenced by family standards. For instance, a ten-year old child may be expected to take care of him or herself without making allowances for the complexity of ART. Families may not understand all the challenges associated with long-term adherence and inaccurately assess the child's capacity for this responsibility. Moreover, personal characteristics or developmental delay may inhibit the child's abilities for adherence. US studies have found a trend toward worse adherence in children who had more responsibility for their own medicines, [15] and better adherence with greater caregiver responsibility for medications.[41]

Family structure may play a role as well. A negative association has been seen between disorganized family structure and adherence.[33] Kinship rules may also be uncertain, especially when the biologic parents are not involved. Certain persons (e.g. the oldest sister of the father) may have a higher authority over the child than the apparent guardian, but do not necessarily participant in clinical care or adherence counseling.[42] Substantial adaptations within the family may therefore be needed to allow for necessary adherence support.

\section{Orphans}

Children who are single or double orphans face particular adherence challenges related to stigma, family structure, access to treatment, and resources. Double orphans in Kenya were found to have worse adherence,[43] but not in Uganda.[20] Loss of one or more parents is often complicated with mental health issues and multiple caregivers, which have been shown to influence adherence as described above.

\section{Poverty and food insecurity}

Many HIV-infected children live in poverty and face food scarcity, both of which have been associated with poor adherence in developing settings.[33,44] Food may be important for the proper dosing of a particular drug, and caregivers may skip doses when food is scarce. Ability to access to ART may lead to inconsistent use and thus poor adherence. Money for transportation to the clinic to pick up ART can be a significant drain on financial resources, as can alternate childcare while the caregiver takes the HIV-infected child or children to clinic. Housing instability has also been associated with non-adherence in US adolescents.[45]

\section{Regimen Characteristics}

Although many advances have been made in decreasing the complexity and pill burden of ART, many challenges remain for children. The following section reviews the challenges faced with the various pediatric drug formulations and frequently changing regimens.

\section{Drug formulation}

Syrups allow for proper dosing in young children; however, they can be difficult to use. They spill easily and caregivers unfamiliar with liquid measurements may not understand how to measure out the precise dose. Elderly caregivers in particular may have difficulty manipulating a syringe, seeing the numbers next to the desired amount, or accurately pouring the liquid into a cup. Additionally, the volume of liquid formulations can be daunting. A young, 15-kg child taking twice daily zidovudine, lamivudine, and nevirapine, for instance, must take a total of $62 \mathrm{~mL}$ of syrup per day. 
Other issues related to syrups are palatability and refrigeration.[28,37] Certain drugs are sweet and easily swallowed. Others, such as ritonavir, are not. Most liquid ART is available in heat stable formulations, although stavudine and ritonavir need refrigeration.

\section{Changing regimens}

In many developing settings, the availability of pediatric ART may be quite limited. Stock outs of a drug entirely, or stock outs of a particular formulation,[34] may require regimen alteration and may lead to confusion. For instance, a given drug may change from a syrup to a tablet or from a combination pill to the individual components. Similarly, as children grow, the dose of each drug must increase, sometimes at every clinical visit. These frequent changes may lead to confusion for the caregiver and child, thus jeopardizing adherence.

\section{Social and Cultural Characteristics}

Many cultures have strong histories of traditional or other forms of non-Western medicine. Literature in adults indicates that use of these medicines for HIV infection is common[46], and cultural norms may pressure caregivers and children to reject ART all together and use alternative medicines in their place. Alternative medicines may also be used in addition to ART, and few pharmacokinetic data is available on traditional and alternative HIV medicines. Adverse drug interactions are therefore a potential concern.

Additionally, in many African countries and in African Americans, there may be a distrust of the Western medicine and/or the medical system. This distrust may stem from unethical medical experiments, such as the Tuskegee Study in which treatment of syphilis was withheld from African Americans to study the natural history of the disease.[47] A US study of HIVinfected adults showed that distrust affected adherence by increasing treatment-related psychological distress and weakening treatment benefit beliefs.[48]

\section{Measurement Tools for Adherence in Children}

The ability to fully understand adherence has been hampered by limitations in the measurement of tools. ART adherence can be measured using multiple methods, which can be categorized as subjective or objective. Subjective measures include provider assessment and child and caregiver self-report. Objective measures consist of pharmacy refill data, announced and unannounced pill counts, and electronic monitoring. There is no gold standard for adherence measurement, and all measures have advantages and disadvantages, depending on individual preferences, available resources, and the goal of clinical care versus research.

Adherence measurement in children is more complicated than measurement in adults due to two principal reasons. First, the goal of the measurement is to learn the behavior of the child; however, that behavior is often influenced to varying degrees by the behavior of one or more caregivers, medical providers, or others. For self-reported measures, the child may be too young to provide information directly and the report must come through the caregiver. Even when the child can provide primary information, studies have shown poor concordance of the data, [15] and the accuracy of both measurements is unclear. Second, most existing adherence measures are designed for adults who take pills with infrequent regimen changes. They do not account for the role of the caregiver(s), the complexity of pediatric regimens, or the measurement of syrups. Objective measures, such as pharmacy refill, that do not depend on these factors are therefore appealing, but do not actually confirm that pills were ingested. Additional research and tools are needed. 


\section{Interventions}

Review of the literature reveals few efficacy-based adherence interventions, most of which were conducted in the US and involve small sample sizes.[7,8] These interventions include directly observed therapy, treatment buddies, stages of change counseling, family therapy, gastrostomy tubes, pill swallowing techniques, and flavoring agents. The lack of comparison groups makes it difficult to assess the impact of the proposed interventions. There has been one randomized controlled trial of a home-based nursing intervention aimed at increasing knowledge and resolving barriers. Additional research is needed for adherence interventions, and lessons may be learned from other pediatric illnesses. A recent meta-analysis of multiple pediatric chronic health conditions found the strongest effect sizes for multi-component and behavioral interventions and those with short term follow-up periods.[49] Weakest effect sizes were seen for instructional interventions and those targeting medications.

\section{Recommendations}

\section{Clinical care}

In spite of limited efficacy-based interventions, several clinical recommendations can be made based on the literature to date. Pediatric adherence to ART is a dynamic process involving the many factors described above. While many clinical programs understandably focus on the provision of medications and management of physical disease, successful ART requires considerable attention to the complex issues of adherence. Recommended approaches must consider the psychosocial aspects of the disease, including child, caregiver and family function, as well as the resources of the child and family. These challenges are especially difficult in settings with few counselors, social workers, and mental health professionals; however, they are now understood to be as important as the drugs themselves.

\section{Research}

Research to date consists largely of studies of adherence estimates using suboptimal adherence measures and correlates of adherence. Although these data are very useful in understanding pediatric ART adherence, research is urgently needed on effective interventions and novel adherence measures that are less affected by subjectivity, changing caregivers, and complex regimens. New measures are also needed to move adherence monitoring from a reactive observation to a potentially proactive intervention.[50] If adherence problems are detected prior to the development of viral rebound, interventions based on the influences discussed in this paper could be applied prior to the loss of treatment efficacy. For example, interventions that promote child and caregiver mental health, family relationships, social support, and caregiver-family relationships may be critical. Additionally, because pediatric ART adherence changes over time and with childhood development, ongoing assessment and support with dynamic, multifaceted interventions will likely be vital to sustained treatment success.

\section{Conclusion}

Adherence behavior in children is more complex than in adults. Efforts to maximize the treatment efficacy in this population must take account of the child, the caregiver(s), the regimens, and influences of the family and society. While much progress has been made in treating HIV-infected children in recent years, especially in developing settings, children have unacceptably high rates of treatment failure and drug resistance. Most children in developing settings have little or no access to second line therapy, and children in developed settings are struggling with chronic treatment. Moreover, many HIV-infected children are living to adolescence and engaging in sexual relationships, which creates a public health risk of 
transmitted resistance. Concerted effort in clinical care and research are urgently needed to support this vulnerable population.

\section{Acknowledgments}

Dr. Haberer's and Mellins' time were supported by grants from the National Institute of Mental Health (K23MH087228 and R01-MH069133, respectively).

\section{References}

1. Joint United Nations Program (UNAIDS) 2008 Report on the global AIDS epidemic; UNAIDS: Geneva. [July 2009]. Available at: http://www.unaids.org/en/Knowledge Centre/HIVData/ GlobalReport/ 2008/2008_Global_report.asp.

2. Burns DN, Mofenson LM. Paediatric HIV-1 infection. Lancet 1999;354(S2):1-6.

3. Wiznia A, Stanley K, Krogstad P, et al. Combination nucleoside analog reverse transcriptase inhibitor (s) plus nevirapine, nelfinavir, or ritonavir in stable antiretroviral therapy-experienced HIV-infected children: week 24 results of a randomized controlled trial--PACTG 377. Pediatric AIDS Clinical Trials Group 377 Study Team. AIDS Res Hum Retroviruses 2000;16:1113-1121. [PubMed: 10954886]

4. Havens, JF.; Mellins, CA. Psychiatric Aspects of HIV/AIDS.. In: Rutter, M.; Bishop, D.; Pine, D.; Scott, S.; Stevenson, J.; Taylor, E.; Thapar, A., editors. Rutter's Child and Adolescet Psychiatry. 5th ed.. Blackwell Publishing; Oxford: 2008. p. 945-955.

5. Yogev R. Balancing the upside and downside of antiretroviral therapy in children. JAMA May 11;2005 293(18):2272-4. [PubMed: 15886383]

6. Scale up of HIV-related prevention, diagnosis, care and treatment for infants and children. World Health Organization; Geneva: 2008 [July 2009]. Available at: http://www.who.int/hiv/pub/paediatric/framework_2008/en/index.html.

7. Vreeman RC, Wiehe SE, Pearce EC, et al. A Systematic Review of Pediatric Adherence to Antiretroviral Therapy in Low- and Middle-Income Countries. Pediatr Infect Dis J 2008;27:686-691. [PubMed: 18574439] [This article provides an excellent summary on pediatric ART adherence studies in developing countries from 1997 to 2007.]

8. Simoni JM, Montgomery A, Martin E, et al. Adherence to Antiretroviral Therapy for Pediatric HIV Infection: A Qualitative Systematic Review With Recommendations for Research and Clinical Management. Pediatrics 2007;119:e1371-83. [PubMed: 17533177] [This article provides a comprehensive overview of studies on pediatric ART adherence from 1996 to 2005.]

9. Zhang F, Haberer J, Wei H, et al. Drug resistance in the Chinese National Pediatric Highly Active Antiretroviral Therapy Cohort: implications for paediatric treatment in the developing world. Int J STD AIDS 2009;20:406-409. [PubMed: 19451326]

10. Smith BA, Shuchman M. Problem of nonadherence in chronically ill adolescents: strategies for assessment and intervention. Curr Opin Pediatr 2005;17:613-618. [PubMed: 16160536]

11. DiMatteo MR. The role of effective communication with children and their families in fostering adherence to pediatric regimens. Patient Educ Couns 2004;55:339-44. [PubMed: 15582339]

12. Centers for Disease Control and Prevention (CDC). HIV/AIDS Surveillance Report: HIV Infection and AIDS in the United States. [July 2009]. Available at: http://www.cdc.gov/hiv/topics/surveillance/resources/reports/2004report/pdf/2004 SurveillanceReport.pdf.

13. Wiener L, Mellins CA, Marhefka S, Battles HB. Disclosure of an HIV diagnosis to children: history, current research, and future directions. J Dev Behav Pediatr 2007;28:155-166. [PubMed: 17435473]

14. Murphy DA, Belzer M, Durako SJ, et al. Longitudinal antiretroviral adherence among adolescents infected with human immunodeficiency virus. Arch Pediatr Adolesc Med 2005;159:764-770. [PubMed: 16061785]

15. Mellins CA, Brackis-Cott E, Dolezal C, et al. The role of psychosocial and family factors in adherence to antiretroviral treatment in human immunodeficiency virus-infected children. Pediatr Infect Dis J 2004;23:1035-1041. [PubMed: 15545859] 
16. Williams PL, Storm D, Montepiedra G, et al. Predictors of adherence to antiretroviral medications in children and adolescents with HIV infection. Pediatrics 2006;118:e1745-57. [PubMed: 17101712]

17. Elise A, France AM, Louise WM, et al. Assessment of adherence to highly active antiretroviral therapy in a cohort of African HIV-infected children in Abidjan, Côte d'Ivoire. J Acquir Immune Defic Syndr 2005;40:498-500. [PubMed: 16280708]

18. Belman MH, Ultmann D, Horoupian B, et al. Neurological complications in infants and children with acquired immune deficiency syndrome. Ann Neurol 1985;18:560-566. [PubMed: 3000281]

19. Brouwer CN, Lok CL, Wolffers I, et al. Psychosocial and economic aspects of HIV/AIDS and counselling of caretakers of HIV-infected children in Uganda. AIDS Care 2000;12:535-540. [PubMed: 11218540]

20. Nabukeera-Barungi N, Kalyesubula I, Kekitiinwa A, et al. Adherence to antiretroviral therapy in children attending Mulago Hospital, Kampala. Ann Trop Paediatr 2007;27:123-131. [PubMed: 17565809]

21. Davies B, Whitsett SF, Bruce A, et al. A typology of fatigue in children with cancer. J Pediatr Oncol Nurs 2002;19:12-21. [PubMed: 11813137]

22. Ene L, Goetghebuer T, Hainaut M, et al. Prevalence of lipodystrophy in HIV-infected children: a cross-sectional study. Eur J Pediatr 2007;166:13-21. [PubMed: 16896646]

23. Adherence to antiretroviral therapy in HIV-infected children and adolescents.; Guidelines for the Working Group on Antiretroviral Therapy and Medical Management of HIV-Infected Children. Feb 232009 [July 2009]. p. 66-72.Available at http://aidsinfo.nih.gov/ContentFiles/PediatricGuidelines.pdf.

24. Feinstein S, Keich R, Becker-Cohen R, et al. Is noncompliance among adolescent renal transplant recipients inevitable? Pediatrics 2005;115:969-973. [PubMed: 15805372]

25. Giacomet V, Albano F, Starace F, et al. Adherence to antiretroviral therapy and its determinants in children with human immunodeficiency virus infection: a multicentre, national study. Acta Paediatr 2003;92:1398-1402. [PubMed: 14971789]

26. Marhefka SL, Tepper VJ, Brown JL, et al. Caregiver psychosocial characteristics and children's adherence to antiretroviral therapy. AIDS Patient Care STDS 2006;20:429-437. [PubMed: 16789856]

27. Biadgilign S, Deribew A, Amberbir A, et al. Adherence to highly active antiretroviral therapy and its correlates among HIV infected pediatric patients in Ethiopia. BMC Pediatr 2008;8:53. [PubMed: 19061515]

28. Van Dyke RB, Lee S, Johnson GM, et al. Reported adherence as a determinant of response to highly active antiretroviral therapy in children who have human immunodeficiency virus infection. Pediatrics 2002;109:e61. [PubMed: 11927734]

29. Bikaako-Kajura W, Luyirika E, Purcell DW, et al. Disclosure of HIV status and adherence to daily drug regimens among HIV-infected children in Uganda. AIDS Behav 2006;10:S85-93. [PubMed: 16791525]

30. Hammami N, Nöstlinger C, Hoerée T, et al. Integrating adherence to highly active antiretroviral therapy into children's daily lives: a qualitative study. Pediatrics 2004;114:e591-597. [PubMed: 15520091]

31. Mellins CA, Brackis-Cott E, Dolezal C, et al. Psychiatric disorders in youth with perinatally acquired human immunodeficiency virus infection. Pediatr Infect Dis J 2006;25:432-437. [PubMed: 16645508]

32. Foster G. Beyond education and food: psychosocial well-being of orphans in Africa. Acta Paediatr 2002;91:502-504. [PubMed: 12113315]

33. Cupsa A, Gheonea C, Bulucea D, et al. Factors with a negative influence on compliance to antiretroviral therapies. Ann N Y Acad Sci 2000;918:351-354. [PubMed: 11131722]

34. Fassinou P, Elenga N, Rouet F, et al. Highly active antiretroviral therapies among HIV-1-infected children in Abidjan, Côte d'Ivoire. AIDS 2004;18:1905-1913. [PubMed: 15353976]

35. Wrubel J, Moskowitz JT, Richards TA, et al. Pediatric adherence: perspectives of mothers of children with HIV. Soc Sci Med 2005;61:2423-2433. [PubMed: 15936134]

36. Nicholson O, Mellins C, Dolezal C, et al. HIV treatment-related knowledge and self-efficacy among caregivers of HIV-infected children. Patient Educ Couns 2006;61:405-410. [PubMed: 16246515] 
37. Reddington C, Cohen J, Baldillo A, et al. Adherence to medication regimens among children with human immunodeficiency virus infection. Pediatr Infect Dis J 2000;19:1148-1153. [PubMed: 11144374]

38. Polisset J, Ametonou F, Arrive E, et al. Correlates of Adherence to Antiretroviral Therapy in HIVInfected Children in Lomé, Togo, West Africa. AIDS Behav 2009;13:23-32. [PubMed: 18654845]

39. Ware NC, Idoko J, Kaaya S, et al. Explaining adherence success in sub-Saharan Africa: an ethnographic study. PLoS Med 2009;6:e11. [PubMed: 19175285]

40. Byrne M, Honig J, Jurgrau A, et al. Achieving adherence with antiretroviral medications for pediatric HIV disease. AIDS Read 2002;12:151-154. 161-164. [PubMed: 12071185]

41. Naar-King S, Montepiedra G, Nichols S, et al. Allocation of family responsibility for illness management in pediatric HIV. J Pediatr Psychol 2009;34:187-194. [PubMed: 18586756]

42. De Baets AJ, Ramet J, Msellati P, et al. The unique features of pediatric HIV-1 in sub-Saharan Africa. Curr HIV Res 2008;6:351-362. [PubMed: 18691033]

43. Vreeman RC, Wiehe SE, Ayaya SO, et al. Association of antiretroviral and clinic adherence with orphan status among HIV-infected children in Western Kenya. J Acquir Immune Defic Syndr 2008;49:163-170. [PubMed: 18769353]

44. Bukusuba J, Kikafunda JK, Whitehead RG. Food security status in households of people living with HIV/AIDS (PLWHA) in a Ugandan urban setting. Br J Nutr 2007;98:211-217. [PubMed: 17381879]

45. Martinez J, Bell D, Camacho R, et al. Adherence to antiviral drug regimens in HIV-infected adolescent patients engaged in care in a comprehensive adolescent and young adult clinic. J Natl Med Assoc 2000;92:55-61. [PubMed: 10800292]

46. Babb DA, Pemba L, Seatlanyane P, et al. Use of traditional medicine by HIV-infected individuals in South Africa in the era of antiretroviral therapy. Psychol Health Med 2007;12:314-320. [PubMed: 17510901]

47. Stebbing J, Bower M. Lessons for HIV from Tuskegee. J HIV Ther 2004;9:50-52. [PubMed: 15534560]

48. Thrasher AD, Earp JA, Golin CE, Zimmer CR. Discrimination, distrust, and racial/ethnic disparities in antiretroviral therapy adherence among a national sample of HIV-infected patients. J Acquir Immune Defic Syndr 2008;49:84-93. [PubMed: 18667919]

49. Kahana S, Drotar D, Frazier T. Meta-analysis of psychological interventions to promote adherence to treatment in pediatric chronic health conditions. J Pediatr Psychol 2008;33:590-611. [PubMed: 18192300]

50. Bangsberg DR. Preventing HIV antiretroviral resistance through better monitoring of treatment adherence. J Infect Dis 2008;197(S3):S272-8. [PubMed: 18447613] 


$$
=
$$

Figure 1.

Factors contributing to pediatric adherence 